\title{
WIENER AND INTEGRATION IN FUNCTION SPACES
}

\author{
BY M. KAC
}

1. Evolution of Mathematics is, by and large, a continuous process and its growth and progress seldom deviate greatly from the natural historical lines. It is because of this that we tend, in retrospect, to admire most those developments which though born well outside it have grown to join and to enrich the mainstream of our science.

It was the great fortune and the great achievement of Norbert Wiener to initiate such a development when, in the early twenties, he introduced a measure, now justly bearing his name, in the space of continuous functions.

2. Let us first review briefly some of the background.

At about 1905, almost simultaneously, and quite independently of each other (in fact, using wholly different approaches) A. Einstein and M. Smoluchowski provided a theory of the peculiar erratic motion of small particles suspended in liquids first described in 1828 by the English botanist Brown.

The theory can be summarized as follows:

(a) For simplicity one confines one's attention to the displacement of the Brownian particle in some chosen direction and one can thus speak of the one-dimensional Brownian motion.

(b) The motion is Markoffian and homogeneous in time; i.e. the probability of finding the particle at times $t_{1}, t_{2}, \cdots, t_{n}\left(0<t_{1}<t_{2}<\right.$ $\left.\cdots<t_{n}\right)$ in the intervals $\left(\alpha_{1}, \beta_{1}\right), \cdots,\left(\alpha_{n}, \beta_{n}\right)$ is given by the formula

$$
\begin{aligned}
& \int_{\alpha_{1}}^{\beta_{1}} \int_{\alpha_{2}}^{\beta_{2}} \cdots \int_{\alpha_{n}}^{\beta_{n}} P\left(x_{0} \mid\right.\left.x_{1} ; t_{1}\right) P\left(x_{1} \mid x_{2} ; t_{2}-t_{1}\right) \cdots \\
& \cdots P\left(x_{n-1} \mid x_{n} ; t_{n}-t_{n-1}\right) d x_{1} \cdots d x_{n}
\end{aligned}
$$

where $P(x \mid y ; t)$ is the probability density of finding the particle at $y$ at time $t$ if it started at $x$ at $t=0$.

(c) For $\Delta t \rightarrow 0$ one has asymptotically

$$
\begin{aligned}
\langle\Delta x\rangle & =\int_{-\infty}^{\infty}\left(x-x_{0}\right) P\left(x_{0} \mid x ; \Delta t\right) d x \sim F\left(x_{0}\right) \Delta t, \\
\left\langle(\Delta x)^{2}\right\rangle & =\int_{-\infty}^{\infty}\left(x-x_{0}\right)^{2} P\left(x_{0} \mid x ; \Delta t\right) d x \sim 2 D \Delta t,
\end{aligned}
$$




$$
\left\langle|\Delta x|^{k}\right\rangle=o(\Delta t), \quad k \geqq 3 .
$$

Here $F(x)$ is the outside force (e.g. gravity) acting on the particle when it is at $x$, and $D$ (the diffusion constant) is given by the formula

$$
D=\frac{2 k T}{f}
$$

where $T$ is the absolute temperature, $f$ the friction coefficient (in liquids e.g. $f$ is given by Stokes' formula $f=6 \pi a \eta$ with $a$ the radius of the spherical particle and $\eta$ the viscosity coefficient) and $k$ the Boltzmann constant.

From (b) it follows at once that $P(x \mid y ; t)$ must satisfy the Smoluchowski equation (often called the Chapman-Kolmogoroff equation),

$$
P(x \mid y ; t+\tau)=\int_{-\infty}^{\infty} P(x \mid \xi ; t) P(\xi \mid y ; \tau) d \xi, \quad \tau>0
$$

and from this, using (c) one can derive (under appropriate smoothness conditions on $P$ ) the diffusion equation

$$
\frac{\partial P}{\partial t}=D \frac{\partial^{2} P}{\partial x^{2}}+\frac{\partial}{\partial x}(F(x) P) .
$$

We have, in addition, the initial condition

$$
P(x \mid y ; t) \rightarrow \delta(y-x), \quad \text { as } t \rightarrow 0,
$$

and the obvious restriction that

$$
P(x \mid y ; t) \geqq 0 \text {. }
$$

If e.g. $F(x)=0$, i.e. we are dealing with a free Brownian particle, the unique solution of the problem is

$$
P(x \mid y ; t)=\frac{1}{2(\pi D t)^{1 / 2}} \exp \left(-\frac{(y-x)^{2}}{4 D t}\right)
$$

and, in particular, the mean-square displacement at time $t$ is given by the formula

$$
\left\langle\Delta^{2}(t)\right\rangle=\int_{-\infty}^{\infty}(y-x)^{2} P(x \mid y ; t) d y=2 D t .
$$

Thus if one observes a large number of free Brownian particles during the same time interval $t$ and equates the empirically calculated mean-square derivation with the theoretically predicted value $2 D t$, 
one can get an estimate of $D$ and hence determine (empirically and approximately) the Avogadro number.

The successful determination of the Avogadro number from Brownian motion experiments was one of the great triumphs of Physics in the early days of the century and it dealt the final blow to the opponents of atomistic theories.

By the time Smoluchowski died in 1917 the theory of Brownian motion was accepted, understood and, in many respects, finished.

3. According to his own account, (see [177]), ${ }^{1}$ Wiener's concern with the problem of measure in function spaces began during his first term as an Instructor at Massachusetts Institute of Technology and it was Professor I. Barnett, of the University of Cincinnati, who drew his attention to the interest of "generalization of the concept of probability to cover probabilities where the various occurrences being studied were not represented by points or dots in a plane or in space but by something of the nature of path curves in space" [177, p. 35].

I find it enormously surprising that, in 1919, when probability theory was not even thought of as a branch of pure mathematics, two young men should have contemplated problems of such degree of sophistication!

Be as it may, Wiener became preoccupied with the subject of measure and integration in a space of curves and in the process he familiarized himself with the work of Gâteaux and especially with that of P. J. Daniell. At the same time he also read G. I. Taylor's pioneering paper on turbulence and he came to think of "the physical possibilities of a theory for averages over curves" [177, p. 37].

"The problem of turbulence," Wiener continues in his autobiography $[177$, pp. $37,38,39]$ "was too complicated for immediate attack, ${ }^{2}$ but there was a related problem which I found to be just right for the theoretical considerations of the field I had chosen for myself. This was the problem of the Brownian motion, and it was to provide the subject of my first major mathematical work. . . . Here I had a situation in which particles describe not only curves but statistical assemblages of curves. It was an ideal proving ground for my ideas concerning the Lebesgue integral in a space of curves, and it had the abundantly physical texture of the work of Gibbs. It was to this field that I had decided to apply the work that I had already done along the lines of integration theory...."

1 The bold-faced numbers in brackets refer to the numbered references in the Bibliography of Norbert Wiener.

It still is today! 
"The Brownian motion was nothing new as an object of study by physicists. There were fundamental papers by Einstein and Smoluchowski that covered it, but whereas these papers concerned what was happening to any given particle at a specific time, or long-time statistics of many particles, they did not concern themselves with the mathematical properties of the curve followed by a single particle.

"Here the literature was very scant, but it did include a telling comment by the French physicist Perrin in his book Les Atomes, where he said in effect that the very irregular curves followed by particles in the Brownian motion, led one to think of the supposed continuous nondifferentiable curves of the mathematicians."

4. Wiener presented his basic ideas in a series of papers published in the period from 1920 to 1923 . He included an account of them in his famous Acta paper on generalized harmonic analysis and he devoted a chapter of his 1934 book with Paley to presenting again the subject to the mathematical community.

The early papers were very difficult to read (as I know from personal experience, when as a student in Lwów I tried to read them with a depressing lack of success) and they fell on deaf ears. Only Paul Lévy in France, who had himself been thinking along similar lines, fully appreciated their significance.

The Acta paper and the book on the Fourier transform in the complex domain were so full of new and exciting results and ideas, which were so unquestionably in the mainstream of analysis that the esoteric "random functions" were somehow overlooked. ing:

The original problem which Wiener posed himself was the follow-

Can one introduce in the space of continuous functions $x(t)(0 \leqq t$ $<\infty, x(0)=0)$ a completely additive measure $\mu$ such that the measure of the set of paths which at times $t_{1}, t_{2}, \cdots, t_{n}\left(0 \leqq t_{1}<t_{2}<\cdots<t_{n}\right)$ pass through the "gates" $\left(\alpha_{1}, \beta_{1}\right),\left(\alpha_{2}, \beta_{2}\right), \cdots,\left(\alpha_{n}, \beta_{n}\right)$ is given by the Einstein-Smoluchowski formula (2.1) with

$$
P(x \mid y ; t)=\frac{1}{(2 \pi t)^{1 / 2}} \exp \left(-\frac{(y-x)^{2}}{2 t}\right) ?
$$

In other words we require that $\mu$ be such that

$$
\begin{aligned}
\mu\left\{\alpha_{1}<x\left(t_{1}\right)<\beta_{1}, \cdots, \alpha_{n}<x\left(t_{n}\right)<\beta_{n}\right\} \\
=\int_{\alpha_{1}}^{\beta_{1}} \cdots \int_{\alpha_{n}}^{\beta_{n}} P\left(0 \mid x_{1} ; t_{1}\right) P\left(x_{1} \mid x_{2} ; t_{2}-t_{1}\right) \cdots \\
\quad \cdots P\left(x_{n-1} \mid x_{n} ; t_{n}-t_{n-1}\right) d x_{1} \cdots d x_{n}
\end{aligned}
$$


with $P$ given by (4.1). (Note that we set $D=\frac{1}{2}$ since the physical nature of the diffusion constant is of no relevance to this phase of the theory.)

Wiener's solution of this problem is embodied in the following theorem:

If $G_{n}, n=0,1,2, \cdots$, is a sequence of independent, normally distributed random variables each having mean 0 and variance 1 , then the series

$$
\frac{G_{0}}{\pi^{1 / 2}} t+\sum_{n=1}^{\infty} \sum_{k=2^{n-1}}^{2^{n}-1} G_{k}\left(\frac{2}{\pi}\right)^{1 / 2} \frac{\sin k t}{k}
$$

converges uniformly in $0 \leqq t<\pi$, with probability 1 , and denoting its sum by $x(t)$ we have (4.2) if we interpret $\mu$ as probability, or equivalently, as the product measure in the product

$$
R^{\infty}=R \times R \times R \times \cdots
$$

with $R$ the real line and the measure $\nu$ in $R$ defined by the formula

$$
\nu(E)=\frac{1}{(2 \pi)^{1 / 2}} \int_{E} \exp \left(-\frac{x^{2}}{2}\right) d x .
$$

Thus to every continuous function $x(t)$ on the interval $0 \leqq t<\pi$ and such that $x(0)=0$ there corresponds a point $\left(G_{0}, G_{1}, G_{2}, \cdots\right)$ in $R^{\infty}$ and conversely, to almost every point in $R^{\infty}$ (in the sense of the product measure defined above) there corresponds a continuous function $x(t)(x(0)=0)$ on $(0, \pi)$. Clearly, the almost one-to-one mapping

$$
x(t) \rightarrow\left(G_{0}, G_{1}, G_{2}, \cdots\right)
$$

can be used to define a measure on the space of continuous functions and while the construction is limited to functions defined on the interval $(0, \pi)$ it can be easily modified to apply to functions defined on $(0, \infty)$.

Having constructed the measure Wiener then proved that almost every $x(t)$ is nowhere differentiable and, even more strongly, that for every $\epsilon>0$ almost every $x(t)$ satisfies the Lipshitz condition with exponent $\frac{1}{2}-\epsilon$ but almost none with exponent $\frac{1}{2}+\epsilon$.

Thus has the remark of Perrin turned into a beautiful theorem!

Let us recall now that at about 1930 there appeared a series of papers by Paley and Zygmund in which, following the lines suggested by the earlier work of Steinhaus and Kolmogoroff and Khintchine, trigonometric series with "random" plus-minus signs

$$
\sum \pm c_{n} \cos n t
$$


were extensively studied. Wiener's results appeared to belong naturally to this circle of problems and indeed Paley and Zygmund joined forces with Wiener in [87] which, in a way, was a "merger" of the two lines of development.

Because of this merger the original motivation was all but lost and what Wiener did took on the appearance of an elegant but special result concerning Fourier series with random coefficients. This subject, by the way, has shown remarkable vitality and there is a significant revival of interest in it largely because of the work of the late Salem and that of Kahane, Katznelson, Malliavin and others.

5. In the meantime measure theoretic foundations of probability theory became firmly established and toward the end of the nineteenthirties it became possible to introduce in a precise and rigorous way very general stochastic processes. This was done mainly by J. L. Doob and while his methods and approach were quite different from (and in some respects preferable to) those of Wiener the basic motivation was, in principle, quite close to Wiener's original one. There were however subtle differences and it is perhaps worthwhile to discuss them briefly especially since in the process we can bring out several other relevant points.

Consider for example the problem of finding the probability that a free Brownian particle starting from $x=0$ will remain to the left of $x=a(a>0)$ for all times $\tau$ not exceeding $t$.

The problem would present no difficulty to a physicist who would, following Smoluchowski, argue as follows.

If a large number of identical particles are started at $x=0$ and watched en masse the macroscopic appearance will be that of classical diffusion; consequently the desired probability is simply the proportion of particles which up to time $t$ did not pass the mark $x=a$. It is thus sufficient to place an absorbing barrier at $x=a$ and calculate the fraction of the diffusing matter to the left of the absorbing barrier at time $t$. This is a classical problem and the answer, as is well known, can be obtained as follows:

Let $Q_{a}(0 \mid x ; t)$ be the fundamental solution of the diffusion equation

$$
\frac{\partial Q}{\partial t}=\frac{1}{2} \frac{\partial^{2} Q}{\partial x^{2}}
$$

subject, in addition to the obvious initial condition

$$
\lim _{t \rightarrow 0} Q(0 \mid x ; t)=\delta(x),
$$


also to the boundary condition

$$
Q(0 \mid a ; t)=0 \text {. }
$$

The desired fraction of the diffusing material is then

$$
q(a ; t)=\int_{-\infty}^{a} Q_{a}(0 \mid x ; t) d x .
$$

Since (for $x \leqq a)$

$$
Q_{a}(0 \mid x ; t)=\frac{1}{(2 \pi t)^{1 / 2}} \exp \left(-\frac{x^{2}}{2 t}\right)-\frac{1}{(2 \pi t)^{1 / 2}} \exp \left(-\frac{(x-2 a)^{2}}{2 t}\right),
$$

we obtain

$$
q(a ; t)=\frac{2}{(2 \pi t)^{1 / 2}} \int_{0}^{a} \exp \left(-\frac{x^{2}}{2 t}\right) d t .
$$

In Wiener's setting the problem is to find the measure of the set of those paths $x(\tau)(x(0)=0)$ which satisfy the condition

$$
x(\tau) \leqq a, \quad 0 \leqq \tau \leqq t .
$$

The explicit representation (4.3) is useless for the purposes of actually computing the measure but since one knows that the paths are continuous, the set defined by (5.6) is measurable and moreover

(5.7) $\mu\{x(\tau) \leqq a ; 0 \leqq \tau \leqq t\}=\lim _{n \rightarrow \infty} \mu\left\{x\left(\frac{k t}{n}\right) \leqq a ; k=1,2, \cdots, n\right\}$.

Using (4.2) we get at once that

$$
\begin{array}{r}
\mu\left\{x\left(\frac{k t}{n}\right) \leqq a ; k=1,2, \cdots, n\right\} \\
=\int_{-\infty}^{a} \cdots \int_{-\infty}^{a} P\left(0 \mid x_{1} ; \frac{t}{n}\right) P\left(x_{1} \mid x_{2} ; \frac{t}{n}\right) \cdots \\
\quad \ldots P\left(x_{n-1} \mid x_{n} ; \frac{t}{n}\right) d x_{1} \cdots d x_{n}
\end{array}
$$

with

$$
P(x \mid y ; t)=\frac{1}{(2 \pi t)^{1 / 2}} \exp \left(-\frac{(y-x)^{2}}{2 t}\right) .
$$

The existence of the limit in (5.7) is a simple consequence of measurability of the set (5.6) and there remains "only" the analytic problem of proving that the limit is indeed equal to $q(a ; t)$. 
In Doob's approach one encounters an interesting difficulty at an earlier stage. Doob begins with the space of all real valued functions $x(\tau)$ (subject to the trivial normalization $x(0)=0$ ) but he keeps $(4.2)$ as the assignment of measures to sets of functions satisfying the conditions

$$
\alpha_{1}<x\left(t_{1}\right)<\beta_{1}, \alpha_{2}<x\left(t_{2}\right)<\beta_{2}, \cdots, \alpha_{n}<x\left(t_{n}\right)<\beta_{n} .
$$

The Borel field generated by sets (5.9) turns out to be too small to make many of the interesting sets measurable. In particular, the set (5.6) is not measurable and neither, for that matter, is the set $C$ of all continuous functions. Fortunately the outer measure of $C$ turns out to be 1 (while the inner measure is 0 ) and because of this it is possible to concentrate the measure on it while maintaining (4.2). In this way one comes back to the Wiener measure but it now emerges as only one of an infinitude of possible measures consistent with (4.2).

The relative delicacy of the situation tended to polarize the interest in stochastic processes along measure-theoretic rather than analytic lines. One might say that more attention was paid to defining measures like

$$
x(\tau) \leqq a ; \quad 0 \leqq \tau \leqq t
$$

than to ways and means of calculating them.

But why fuss over measure-theoretic points when all the time the answer could be obtained (as shown above) by a simple application of classical diffusion theory?

The answer lies somewhat deeper than one might think.

It can be verified by an elementary computation that (5.4) and (5.5) imply for $x \leqq a$ the equation

$$
Q_{a}(x ; t)=P(0 \mid x ; t)-\int_{0}^{t}\left(-\frac{\partial q(a ; \tau)}{\partial \tau}\right) P(a \mid x ; t-\tau) d \tau
$$

or equivalently

$$
\begin{array}{r}
\int_{I} Q_{a}(x ; t) d x+\int_{0}^{t}\left(-\frac{\partial q(a ; \tau)}{\partial \tau}\right) \int_{I} P(a \mid x ; t-\tau) d x d \tau \\
=\int_{I} P(0 \mid x ; t) d x
\end{array}
$$

where $I$ is an interval to the left of $x=a$.

Note now that

$$
-\frac{\partial q(a ; \tau)}{\partial \tau} d \tau
$$


is the (differential) probability that the Brownian path $x(t)(x(0)=0)$ will leave the half line $x \leqq a$, for the first time, between $\tau$ and $\tau+d \tau$, and consequently

$$
-\frac{\partial q(a ; \tau)}{\partial \tau} d \tau \int_{I} P(a \mid x ; t-\tau) d x
$$

is the probability that the path will end up in $I$ at time $t$ having crossed $x=a$ for the first time between $\tau$ and $\tau+d \tau$. The second integral on the right hand side of (5.12) is thus the probability of ending up in $I$ at time $t$ having left $(-\infty, a)$ sometime before, and the first integral is clearly the probability of ending up in $I$ at time $t$ without having left that half line; together they add up to the probability of ending up in $I$ at time $t$ and equation (5.12) follows.

But in arriving at this appealing interpretation I have identified leaving $(-\infty, a)$ and crossing $x=a$; this identification is justified only if the paths are continuous and we are again led to the question of continuity of paths!

To appreciate this more fully let us imagine for a moment that the displacement of a particle was governed by the Cauchy density

$$
P(x \mid y ; t)=\frac{t}{\pi} \frac{1}{t^{2}+(x-y)^{2}}
$$

instead of the Gaussian density (4.1).

This density also satisfies the Smoluchowski equation (2.2) and hence it is consistent to use formula (4.1) to assign measures to sets (5.9).

Probability $q(a ; t)$ could still be defined by formulas (5.7) and (5.8) with $P$ given by $(5.13)$ and $Q_{a}(x ; t)$ can be defined by the formula

$$
\begin{array}{r}
Q_{a}(x ; t)=\lim _{n \rightarrow \infty} \int_{-\infty}^{a} \cdots \int_{-\infty}^{a} P\left(0 \mid x_{1} ; \frac{t}{n}\right) P\left(x_{2} \mid x_{3} ; \frac{t}{n}\right) \cdots \\
\cdots P\left(x_{n-1} \mid x ; \frac{t}{n}\right) d x_{1} \cdots d x_{n-1}
\end{array}
$$

again with $P$ being the Cauchy density (5.13). The limits involved in (5.7) and (5.14) can be shown to exist and we might think that the analogy with the Gaussian case is so complete that (5.11) also holds. This however is not so!

The reason is that no longer can the measure be concentrated on the space of continuous functions and therefore "leaving $(-\infty, a)$ " and "crossing $x=a$ " are not the same. In fact, because of the discontinu- 
ous nature of the paths "crossing $x=a$ " is not even properly defined.

That the the sample paths of the Cauchy process (and other socalled stable processes) cannot be continuous (i.e. assigning the measure (4.1) with $P$ given by (5.13) to sets (5.9) makes it impossible to concentrate the measure on the space $C$ of continuous functions ${ }^{3}$ ) was first noted by Paul Lévy who has also shown that the sample paths, though by necessity discontinuous, can be made right (or left) continuous. ${ }^{4}$ This development helped to underscore the depth of Wiener's achievement although full awareness of capabilities and potentialities of measure and integration theories in function spaces was rather slow in coming.

6. During the war years there has been a substantial increase of interest in stochastic processes owing mainly to the need of analyzing noise phenomena in radar and related electronic systems.

Here methods based on trigonometric series with random coefficients are especially appealing and natural.

Wiener always had a strong interest in circuits and in other phases of electrical engineering and he continued almost to the end of his life to relate his early work on "random functions" to linear and nonlinear problems in circuit theory (see e.g. [191]).

But the strongest impact of Wiener's ideas on mathematics came from different directions.

Starting in 1943, R. H. Cameron and W. T. Martin published a series of papers devoted to the calculation of Wiener integrals of a class of functionals.

Their work culminated in proving that if $p(t) \geqq 0,0 \leqq t \leqq 1$, is continuous, then the Wiener integral of

$$
\exp \left(\lambda \int_{0}^{1} p(t) x^{2}(t) d t\right)
$$

is equal, for sufficiently small $\lambda$, to

$$
\prod_{k=1}^{\infty} \frac{1}{\left(1-\lambda \lambda_{k}\right)^{1 / 2}}
$$

where the $\lambda_{k}$ 's are the eigenvalues of the Sturm-Lionville problem

$$
\phi^{\prime \prime}+\frac{1}{\lambda} p(t) \phi=0, \quad \phi(0)=\phi^{\prime}(0)=0 .
$$

\footnotetext{
3 In fact, in Doob's formulation described above, the outer measure of $C$ is 0 .

4 Doob has greatly extended and systematized this work.
} 
To derive this result Cameron and Martin developed an interesting theory of linear "changes of variable" in Wiener space in which linear integral equations played an important part.

This was the first inkling that there are highly nontrivial connections between classical analysis and integration in function spaces.

Almost at the same time Kakutani published a series of notes on connections between potential theory and Wiener measure. Of his results perhaps the most important was one identifying sets of capacity zero with those which Brownian motion curves hit with probability zero. This was a beginning of an extensive and fruitful development which continues to this day.

Then in 1948 there appeared in print a part of the 1942 doctoral dissertation of R. P. Feynman which dealt with a highly suggestive reformulation of the nonrelativistic quantum mechanics in terms of a certain integral over the space of paths.

To review briefly what is at stake let us restrict ourselves to the case of a particle of mass $m$ moving along the $x$-axis in a force field generated by the potential $V(x)$.

The basic problem is to find the probability that if at time 0 the system is in the state described by the wave function $\phi_{0}$, it will be in the state described by the wave function $\phi_{1}$ at time $t$. This probability is of the general form

$$
\left|\left\langle\phi_{0}|K| \phi_{1}\right\rangle\right|^{2}
$$

where $K$ is an appropriate operator. In our one-dimensional case,

$$
\left\langle\phi_{0}|K| \phi_{1}\right\rangle=\int_{-\infty}^{\infty} \int_{-\infty}^{\infty} \phi_{0}\left(x_{0}\right) K\left(x_{0} \mid x_{2} ; t\right) \phi_{1}(x) d x_{0} d x
$$

where the "propagator" $K\left(x_{0} \mid x ; t\right)$ is obtained by solving the Schroedinger equation

$$
-\frac{\hbar}{i} \frac{\partial K}{\partial t}=-\frac{\hbar^{2}}{2 m} \frac{\partial^{2} K}{\partial x^{2}}+V(x) K
$$

subject to the initial condition

$$
K\left(x_{0} \mid x ; t\right) \rightarrow \delta\left(x-x_{0}\right) .
$$

What Feynman noted was that $K$ can be formally written as a certain average over the set of all paths $x(\tau)$ such that 


$$
x(0)=x_{0}, \quad x(t)=x
$$

of the quantity

$$
\exp \left(\frac{i}{\hbar} S[x]\right)
$$

where

$$
S[x]=\int_{0}^{t}\left\{\frac{m}{2}\left(\frac{d x}{d \tau}\right)^{2}-V(x(\tau))\right\} d \tau
$$

is the classical action integral (the integrand is the Lagrangean of the system). Symbolically,

$$
K\left(x_{0} \mid x ; t\right)=\int \exp \left(\frac{i}{\hbar} S[x]\right) d \text { (path). }
$$

Feynman proposed to define his "integral" (6.11) as the limit

$$
\lim _{n \rightarrow \infty} \frac{\int_{-\infty}^{\infty} \cdots \int_{-\infty}^{\infty} \exp \left[\frac{i}{\hbar} \Delta_{n} \sum_{k=0}^{n-1}\left\{\left(\frac{m}{2}\left(\frac{x_{k+1}-x_{k}}{\Delta_{n}}\right)\right)^{2}-V\left(x_{k}\right)\right\}\right] d x_{1} d x_{2} \cdots d x_{n-1}}{\int_{-\infty}^{\infty} \cdots \int_{-\infty}^{\infty} \exp \left[\frac{i}{\hbar} \Delta_{n} \sum_{k=0}^{n-1} \frac{m}{2}\left(\frac{x_{k+1}-x_{k}}{\Delta_{n}}\right)\right] d x_{1} d x_{2} \cdots d x_{n-1}}
$$

where $\Delta_{n}=t / n$, and $x_{n}=x$. Leaving aside questions of convergence he then showed (formally) that if $K$ is defined in this way it is indeed the correct propagator i.e. it satisfies (6.6) and (6.7).

In 1947 Feynman and I were colleagues at Cornell and I attended a lecture of his at the Physics Colloquium at which he presented his formulation of quantum mechanics. At the time I happened to be interested in calculating various Wiener integrals (some suggested by discussions with W. T. Martin to whom I owe a great deal for getting me involved with the subject of integration in function spaces) and as I sat listening to Feynman it occurred to me that if instead of (6.6) one were to take the real heat equation

$$
\frac{\partial Q}{\partial t}=\frac{1}{2} \frac{\partial^{2} Q}{\partial x^{2}}-V(x) Q
$$

(still subject to the initial condition $Q\left(x_{0} \mid x, t\right) \rightarrow \delta\left(x-x_{0}\right)$ as $\left.t \rightarrow 0\right)$, then one could write its solution (Green's function) as an appropriate Wiener integral. 
In fact, if $V(x)$ is bounded from below and satisfies mild regularity conditions and if $f(x)$ is integrable, then

$$
\begin{aligned}
& \int_{-\infty}^{\infty} Q\left(x_{0} \mid x ; t\right) f(x) d x \\
& =E\left\{\exp \left(-\int_{0}^{t} V\left(x_{0}+x(\tau)\right) d \tau\right) f\left(x_{0}+x(t)\right)\right\}
\end{aligned}
$$

where $E\{\cdots\}$ denotes the Wiener integral of the functional inside the braces. $Q$ itself could be obtained either by an appropriate limit process or, equivalently, by considering conditional Wiener integrals ${ }^{5}$ (expectations).

The formula then is

$$
\begin{aligned}
& Q\left(x_{0} \mid x ; t\right)=\frac{\exp \left(-\frac{\left(x-x_{0}\right)^{2}}{2 t}\right)}{(2 \pi t)^{1 / 2}} \\
& \cdot E\left\{\exp \left(-\int_{0}^{t} V\left(x_{0}+x(\tau)\right) d \tau\right) \mid x(t)=x-x_{0}\right\} .
\end{aligned}
$$

If one were to follow Feynman, one would try to define $Q$ (in analogy with (6.12)) as the limit

$$
\lim _{n \rightarrow \infty} \frac{\int_{-\infty}^{\infty} \cdots \int_{-\infty}^{\infty} \exp \left[-\Delta_{n} \sum_{k=0}^{n-1}\left\{\frac{1}{2}\left(\frac{x_{k+1}-x_{k}}{\Delta_{n}}\right)^{2}+V\left(x_{k}\right)\right\}\right] d x_{1} \cdots d x_{n-1}}{\int_{-\infty}^{\infty} \cdots \int_{-\infty}^{\infty} \exp \left[-\Delta_{n} \sum_{k=0}^{n-1} \frac{1}{2}\left(\frac{x_{k+1}-x_{k}}{\Delta_{n}}\right)^{2}\right] d x_{1} \cdots d x_{n-1}}
$$

but now, owing to the rigorously established existence of the Wiener measure, ${ }^{6}$ the existence of the limit is an immediate consequence of the measurability of the functional

$$
\int_{0}^{t} V\left(x_{0}+x(\tau)\right) d \tau
$$

The fact that one can relate solutions of differential equations to function space integrals suggested many directions of further work. By now the literature on the subject is so vast and so varied that even a cursory review would be out of the question. In some instances ex-

${ }_{5}^{5}$ This amounts to constructing a Wiener like measure in the set of continuous paths $y(\tau)$ such that $y(0)=0$ and $y(t)=y$. The simplest way to accomplish this is to map the ordinary Wiener measure in space of paths $x(\tau)(x(0)=0)$ by means of the mapping $y(\tau)=x(\tau)+(\tau / t)(y-x(t)), 0 \leq \tau \leq t$.

${ }^{6}$ More precisely, the Wiener like measure described in the preceding footnote. 
treme generality helps hide the true origins in Wiener's measure and integration, and one is reminded of a statement attributed (apocryphally perhaps) to Hilbert that the art of doing mathematics consists in finding that special case which contains all the germs of generality.

Feynman's ideas contributed greatly to gaining a larger audience for the work based on the Wiener integral and helped to take the "Wiener process" out of the somewhat narrow context of stochastic processes and into a much wider stream of analysis and physics.

As an illustration let me describe a simple application of the Wiener integral to quantum statistical mechanics.

The quantum-mechanical partition function is defined as the sum

$$
Z=\sum_{n=1}^{\infty} e^{-\beta E_{n}},
$$

where the $E_{n}$ 's are the eigenvalues of the Schroedinger operator

$$
-\frac{\hbar^{2}}{2 m} D^{2}+V(x) \quad\left(D \equiv \frac{d}{d x}\right)
$$

and

$$
\beta=\frac{1}{k T}
$$

with $T$ the absolute temperature and $k$ the Boltzmann constant. It can be easily related to the so called Bloch equation

$$
\frac{\partial P}{\partial \beta}=\frac{\hbar^{2}}{2 m} \frac{\partial^{2} P}{\partial \beta^{2}}-V(x) P
$$

which, except for units, is of the "real" heat equation (6.13). It was, of course, well known that

$$
Z=\int_{-\infty}^{\infty} P(x \mid x ; \beta) d x
$$

where $P\left(x_{0} \mid x ; \beta\right)$ is the Green's function of (6.19). But now, owing to (6.15), one could also write $Z$ as a function space integral and in fact,

$$
\begin{aligned}
Z= & \frac{m^{1 / 2}}{\hbar} \frac{1}{(2 \pi \beta)^{1 / 2}} \\
& \cdot \int_{-\infty}^{\infty} E\left\{\exp \left(-\int_{0}^{\beta} V\left(x+\frac{\hbar}{m^{1 / 2}} x(\tau)\right) d \tau\right) \mid x(\beta)=0\right\} d x .
\end{aligned}
$$


To appreciate the advantage resulting from the use of function space integrals consider the classical limit $h \rightarrow 0$.

It is now absolutely transparent what to expect.

Since as $h \rightarrow 0$

$$
E\left\{\exp \left(-\int_{0}^{\beta} V\left(x+\frac{\hbar}{m^{1 / 2}} x(\tau)\right) d \tau\right) \mid x(\beta)=0\right\} \rightarrow \exp (-\beta V(x)),
$$

we have formally

$$
\begin{aligned}
\lim _{h \rightarrow 0}(\hbar Z) & =\frac{m^{1 / 2}}{(2 \pi \beta)^{1 / 2}} \int_{-\infty}^{\infty} \exp (-\beta V(x)) d x \\
& =\int_{-\infty}^{\infty} \exp \left(-\beta\left(\frac{p^{2}}{2 m}\right)\right) d p \int_{-\infty}^{\infty} \exp (-\beta V(x)) d x \\
& =\int_{-\infty}^{\infty} \int_{-\infty}^{\infty} \exp \left(-\beta\left(\frac{p^{2}}{2 m}+V(x)\right)\right) d x d p
\end{aligned}
$$

which is the classical partition function since

$$
\frac{p^{2}}{2 m}+V(x)
$$

is the Hamiltonian of the system.

The formal steps can be justified at the expense of some mild conditions on $V$. Under appropriately stronger conditions on $V(6.21)$ can be made a starting point (as was, in fact, done by Siegert and Yaglom) of a simple and straightforward derivation of the KirkwoodWigner expansion of $Z$ in powers of $h$.

While on the subject of quantum mechanics one should at least mention another line of development of integration in function spaces. This has to do with an integration theory in Hilbert space and was dictated by certain needs of quantum theory of fields. Originated by Friedrichs and I. E. Segal this theory has, more recently, been shown to be closely related to and, in some respects, equivalent with Wiener's integration theory in the space of continuous functions. As one example of the interplay of the two "schools of thought" we may mention e.g. L. Gross' Hilbert space version of the striking inversion formula of Cameron and Donsker which they discovered and proved in the context of Wiener integrals.

Also the Cameron-Martin theory of linear changes of variable alluded to above assumes a particularly appealing form in Hilbert space setting. 
7. Wiener did not directly participate in the evolution described above and in fact, the only reference to it in his autobiography [177] is the brief statement (pp. 179-180) that "He (Martin) and Cameron did a good deal of work together along the lines of my Brownian motion papers, and they organized the field into a generally recognized branch of mathematical work."

Like all highly creative and highly original people Wiener preferred thinking to reading and he did not take the trouble of familiarizing himself with the rapidly growing body of literature. But he had (and justly so!) a strong proprietory feeling toward his integral and he watched the work related to it closely enough to raise a few questions of priority.

What is perhaps really surprising is that he was apparently unaware of the intimate connections between his measure and his own work on potential theory.

It is now almost universally known that the generalized WienerPerron capacitory potential of a closed set $F$ (in Euclidean space of dimension 3 or higher) at a point $p$ is the Wiener measure of the set of paths which originate from $p$ and which at some time hit $F$. Moreover, Wiener's famous criterion for regularity of boundary points has a most appealing interpretation in terms of his measure. ${ }^{7}$

The surprise is heightened if one recalls that Wiener's work on potential theory was almost simultaneous with that on Brownian motion.

But while Wiener did not take active part in most of the probabilistic and analytic developments which his early work inspired his interest in the preoccupation with random functions always remained strong.

I have already mentioned his book [191].

One should also mention his (somewhat obscurely motivated) paper on Homogeneous Chaos [108] which, more recently, in the hands of Ito, Kakutani and Segal, has found interesting uses in the theory of unitary operators and in tensor algebras over Hilbert spaces.

Finally, there was the attempt to use randon functions to achieve a sort of "hidden variables" interpretation of quantum mechanical probabilities. This was first discussed in the paper [167] written jointly with Armand Siegel and in spite of a rather indifferent reception it received, Wiener had high hopes for this approach. It was taken up again in [209] which is probably his last published paper.

${ }^{7}$ A very complete discussion of this subject can be found in the recent book of Ito and McKean Diffusion processes and their sample paths, Springer-Verlag, New York. 
8. In retrospect one can have nothing but admiration for the vision which Wiener had shown when, almost half a century ago, he had chosen Brownian motion as a subject of study from the point of view of the theory of integration. To have foreseen, at that time, that an impressive edifice could be erected in such an esoteric corner of mathematics was a feat of intuition not easily equalled now or ever.

It was Josiah Willard Gibbs, whom Wiener admired so much who said that "one of the principal objects of the theoretical research in any department of knowledge is to find the point of view from which the subject appears in its greatest simplicity."

Integration in function spaces provided such a point of view over and over again in widely scattered areas of knowledge and it gave us not only a new way of looking at problems but actually a new way of thinking about them.

The fate of all great work is to be subsumed; the more attention it attracts the greater the chances of becoming engulfed in a cascade of generalizations and extensions.

This is especially true today because of a growing tendency to believe that the latest improvement supersedes all that preceded it and that a generalization constitutes a license to subsume.

It is therefore well to repeat that Wiener's contribution to the subject of integration in function spaces will forever be the greatest because he had the idea first; and should anyone try to attribute it to luck let him be reminded that it is the deserving ones who are also lucky.

The Rockefeller University 